\title{
Astrophysical outflows simulated by laser-driven plasma jets
}

\author{
C. Michaut ${ }^{1}$, C. D. Gregory ${ }^{2}$, B. Loupias ${ }^{3}$, E. Falize ${ }^{1,3}$, A. Ravasio ${ }^{2}$, \\ A. Dizière ${ }^{2}$, T. Vinci ${ }^{2}$, M. Koenig ${ }^{2}$ and S. Bouquet ${ }^{1,3}$ \\ ${ }^{1}$ LUTH, Observatoire de Paris, CNRS, Université Paris-Diderot, 92190 Meudon, France \\ email: claire.michaut@obspm.fr \\ ${ }^{2}$ LULI, CNRS, CEA, Université Paris VI, Ecole Polytechnique, 91128 Palaiseau, France \\ ${ }^{3}$ CEA-DAM-DIF, F-91297 Arpajon, France
}

\begin{abstract}
Within the framework of laboratory astrophysics, we form a qualified multidisciplinary group in radiative hydrodynamics. Since 10 years, we have developed laboratory experiments as radiative shocks and plasma jets in connection to astrophysics. Such laboratory experiments provide a unique opportunity to validate models and numerical schemes introduced in radiative hydrodynamics codes. Here we summarize our experimental researches about plasma jets. Laboratory astrophysical experiments have been performed using LULI2000 (France), VULCAN (UK) and GEKKO XII (Japan) intense lasers. The goal of these experiments is to investigate some of the complex features of jets from Young Stellar Objects (YSO), and in particular its interaction with the interstellar medium (ISM).
\end{abstract}

Keywords. jet, laboratory astrophysics, radiative hydrodynamics, laser-plasma interaction

\section{Introduction}

For studying YSO jet propagation, experimental research related to laboratory astrophysics using an intense laser were performed. The relevance of these experiments to astrophysics is measured through scaling laws (Falize et al. 2008, Bouquet et al. 2010). They ensure the similarity between astrophysics and laboratory provided that the dimensionless numbers are equivalent. These non-exhaustive numbers are Mach $M$, Reynolds $R e$, Peclet $P e$ and Mihalas $R$ numbers, the cooling coefficient $\chi$, the aspect ratio and the jet-to-ambient density ratio $\eta$. Some of these numbers are estimated at the time of the experiment design while others are measured during the experiment. The range of values for these parameters represent upper and lower limits given by the accuracy of the experimental measurements which are thus crucial. An important number of temporallyresolved optical diagnostics are implemented and in some experiments, X-ray radiography and proton shadowgraphy are added. X-rays are able to diagnose the dense plasma part, but the more tenuous regions are below the diagnostic sensitivity. In contrast, the optical probe is unable to penetrate high-density jet cores, but is well suited to imaging relatively low plasma densities. Proton radiography is able to image small-scale density structures in the dense parts with high resolution. The capability of fielding these diagnostics on a single shot is beneficial, and allows a more complete understanding of the jet dynamics. We designed different targets: metallic V-foils, foam cones and conical metallic shells. A jet-like structure is observed and its time evolution is studied by varying either the foam density or the metal nature. Nominally identical jets were propagated into vacuum. The interaction with ambient medium is realized using a gas outlet nozzle ensuring a ratio $\eta$ close to astrophysical conditions $(2 \leqslant \eta \leqslant 50)$. 


\section{Summarized Experimental Results}

At the beginning, a serie of experiments was driven by using V-foil targets irradiated by two laser beams. The thin aluminum foils explode and collide, driving a narrow plasma flow. The major results are presented in Gregory et al. (2008), (2009). The jet propagating in gas has a reduced width when compared to the vacuum case, and shock structures appears at the leading edge of the flow. This is consistent with numerical simulations indicating that an ambient medium should increase the collimation of laser produced jets. A velocity was recorded around $300 \mathrm{~km} / \mathrm{s}$ and a temperature of $10 \mathrm{eV}$ was measured for these flows. Dimensionless numbers are satisfied, but as these targets have no cylindrical geometry, the jet is not cylindrical. These preliminary experiments allowed to determine the optimal irradiated angle to obtain a good collimation and to design cone targets.

Numerical simulations (Vinci et al. 2008) allowing to understand the collimated plasma generation have permit to design an original target using a cone filled with foam. In this case the larger cone side is irradiated and a collimated plasma is ejected by the smaller side. The full experimental set-up and results are readable in Loupias et al. (2007a), (2007b), (2009a), Gregory et al. (2010a). The jet velocity ( [90-190] km/s) into vacuum accords with analytical predictions (Falize et al. 2008b), and slows down of 10\% in ambient medium due to the bow shock creation. The measured envelope temperature is $2 \mathrm{eV}$ since the estimated central one is $15 \mathrm{eV}$. The cocoon is clearly shown comparing visible and X-ray diagnostics, but it has not a large aspect ratio instead of the highcollimated dense core. In addition, the proton radiography shows a perturbed shape of the interface jet/ambient medium which is not yet explained. Physical reasons lead to the fact this interface is Rayleigh-Taylor unstable. As the foam is a hydrogen/carbon mixture, it gives a jet more scalable to astrophysical outflow with dimensionless numbers displaying good similarities except for $\chi$ showing the radiation escaping is too low.

An third target type is used to investigate the radiative cooling effects on the jet morphology. Jets are created through laser irradiation of thin conical shells of either gold, copper or aluminum. In choosing different atomic numbers, the degree of radiative losses is altered and plays a role on collimation. The data in Gregory et al. (2010b) show the importance of the conical geometry creating high-collimated outflow. The results suggest the jet collimation is increased for higher-atomic number targets and are consistent with computer simulations showing a radial collapse. Conical shells give the better collimated jet but their temperature is not very well known, therefore the radiative cooling can not be estimated.

Roughly these laboratory experiments are in a regime scalable to YSO jets.

\section{References}

Bouquet, S., Falize, E., Michaut, C., Gregory, C. D., and 3 coauthors, 2010, HEDP doi:10.1016/j.hedp.2010.03.001

Falize, E., Bouquet, S., Michaut, C., 2008a, JPCS, 112, 042015

Falize, E., Bouquet, S., Michaut, C., 2009, Ap\&SS, 322, 107

Gregory, C. D., Loupias, B., Waugh, J., Barroso, P., and 20 coauthors, 2008, PPCF, 50, 124039

Gregory, C. D., Howe, J., Loupias, B., Myers, S., and 9 coauthors, 2009, Ap\&SSS, 322, 37

Gregory, C. D., Loupias, B., Waugh, J., Dono, S., and 9 coauthors, 2010a, PoP, 17, 052708

Gregory, C. D., Dizière, A., Aoki, H., Besio, M., and 13 coauthors, 2010b, submitted in ApESSS

Loupias, B., Koenig, M., Falize, E., Bouquet, and 10 coauthors, 2007a, PRL 99, 265001

Loupias, B., Falize, E., Koenig, M., Bouquet, S., and 9 coauthors, 2007b, Ap\&SS, 307, 103

Loupias B., Falize E., Gregory C. D., Vinci, T., and 14 coauthors, 2009a, PPCF, 51, 124027

Loupias, B., Gregory, C. D., Falize, E., Waugh and 13 coauthors, 2009b, Ap\&SSS, 322, 25

Vinci, T., Loupias B., Koenig, M., Benuzzi-Mounaix, and 7 coauthors, 2008, JPCF, 112, 042012 